\title{
Proximal Probes Based Nanorobotic Drawing of Polymer Micro/Nanofibers
}

\author{
Amrinder Singh Nain, Cristina Amon, Fellow, IEEE, and Metin Sitti, Member, IEEE
}

\begin{abstract}
This paper proposes a nanorobotic fiber fabrication method which uses proximal probes to draw polymer fibers down to few hundred nanometers in diameter and several hundred micrometers in length. Using proximal probes such as Atomic Force Microscope (AFM) and Scanning Tunneling Microscope (STM) or glass micropipettes, liquid polymers dissolved in a solvent are drawn. During drawing, the solvent evaporates in real-time which solidifies the fiber. Controlling the drawn fibers trajectory and solidification in three-dimensions (3-D), suspended fibers, fiber cantilevers, custom 3-D fibers, and fiber networks, are proposed to be fabricated. Poly(methyl methacrylate) (PMMA) polymer dissolved in chlorobenzene is used to form a variety of suspended polymer fibers with diameters from few microns to $200 \mathrm{~nm}$. Fabrication of crossed and linear networks of fibers is also demonstrated. Viscoelastic modeling of polymer fiber drawing is realized using a finite element method to test the significance of the drawing speed and velocity profile on the extensional behavior of the drawn fiber. Since the mechanical properties of the drawn micro/nanofibers could vary from the bulk polymer material significantly, mechanical characterization of suspended fibers using an AFM and a Nanoindenter setup is proposed. Extending this technique to a variety of nonconductive and electroactive polymer fibers, many novel applications in micro/nanoscale sensors, actuators, fibrillar structures, and optical and electronic devices would become possible.
\end{abstract}

Index Terms-Nanomanipulation, nanorobotics, polymer micro/ nanofibers, proximal probes.

\section{INTRODUCTION}

$\mathbf{F}$ ABRICATION of polymer fibers with diameters ranging from micron to nanometer scale has generated significant interest due to its potential impact in many applications such as nano-electronics [1] and optical sensors [2]. These applications require customized three-dimensional (3-D) framework of nanofibers, which when taken individually, form the one-dimensional (1-D) nanoscale building blocks in bottom-up assembly manufacturing process. This hierarchical approach first creates the individual components and then assembles them together into larger structures. The main target of this paper is to identify a novel nanorobotic method of fabricating 3-D polymer micro/nanofibers using different proximal probe-based drawing

Manuscript received February 28, 2006; revised May 3, 2006. This work was supported in part by the Pennsylvania Department of Community and Economic Development under a PITA Grant and in part by the National Science Foundation (NSF) through the NSF CAREER Award Program under Grant NSF IIS0448042.. The review of this paper was arranged by Associate Editor T. Fukuda.

The authors are with the Department of Mechanical Engineering and Institute for Complex Engineered Systems (ICES), Carnegie Mellon University, Pittsburgh, PA 15213 USA (e-mail: anain@ andrew.cmu.edu; camon@andrew.cmu. edu; msitti@andrew.cmu.edu).

Color versions of Figs. 1, 3-12, 16, 19, 22, 24, and 25 are available online at http://ieeexplore.ieee.org.

Digital Object Identifier 10.1109/TNANO.2006.880453
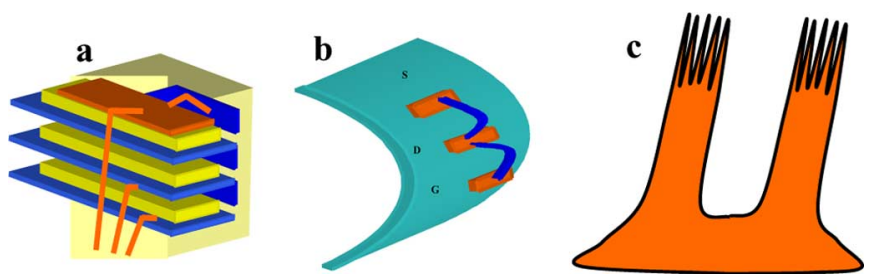

Fig. 1. Polymer micro/nanofibers applications: (a) interconnects in vertical integrated circuit chip packaging, (b) interconnects on flexible substrates, (c) biomimetic fibrillar adhesives.

techniques. This paper further draws insights from finite element simulations into the effects of key automation parameters on fabricating submicron high aspect polymer fibers. Finally, this paper aims at mechanically characterizing the suspended fibers obtained and addressing the open issues.

Current fiber fabrication techniques deposit or fabricate fibers essentially in one dimension, whereby a fiber or series of fibers are deposited and aligned in one step and have limited applications. Customized 3-D fabrication of individual polymer micro and nanoscale fibers has wide ranging applications and the potential to introduce and mature new and existing novel technologies as shown in Fig. 1. Conductive polymers can be used as electrical interconnects [3], [4] and nonconductive fibers can also be used as mechanical elements in micro-electro-mechanical systems (MEMS) or as biomimetic fibrillar adhesives [5]. In addition, fabricating fibers using proximal probes can be used to study the rheological behavior of polymers at the submicron scales [6], [7]. Furthermore, nanoelectronics envisions that 3-D electrical connections will be made where nanowires are orthogonal or overlap. In such a design a crossbar array or a mesh of wires can serve as a basic repeated unit [1]. Creating such networks involves depositing fibers in layers, in which each new layer is orthogonal or at some desired orientation to the previously deposited layer. The classification of the fiber arrangements is outlined in Fig. 2, where free standing and highly oriented fibers are drawn with different pitch separations and diameters in one, two and three dimensions.

The most common process of fabricating continuous polymer fibers ranging from tens of nanometers to one micrometer in diameter is by electrospinning [8]-[14]. Electrospinning is based on the uniaxial stretching of a viscoelastic jet derived from a polymer solution or melt. Due to the chaotic motion of the highly charged jet in electrospinning, the fibers are often collected as randomly oriented structures in the form of nonwoven mats, and as such, have limited applications. A number of schemes have been proposed [15]-[21] for aligning electrospun polymer fibers. The major challenges [12] encountered in the electrospinning process are the uniformity of fiber 


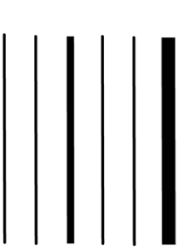

(a)

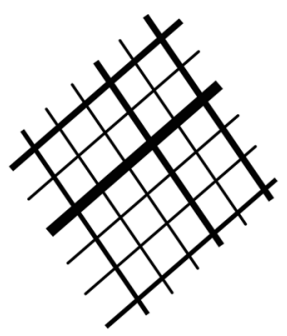

(b)

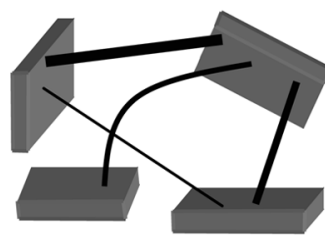

(c)
Fig. 2. Classification of fiber arrangements in one (a), two (b), and three (c) dimensions with different pitch separations and diameters. (a) One-dimensional fibers; (b) two-dimensional arrays; (c) three-dimensional fibers.

diameter, defects such as beads, and nonalignment of fibers upon deposition.

Another method for fabricating fibers, which is similar to dry-spinning in the industry, is drawing. Drawing a fiber requires a material with a pronounced viscoelastic behavior to undergo strong deformations, while being cohesive enough to support the stresses developed during the pulling. The drawing process is always accompanied by a solidification that transforms the drawn fiber into a solid fiber. Polymeric micro/nanofibers have been fabricated using glass micro-pipettes [22] and atomic force microscope (AFM) or scanning tunneling microscope (STM) probe tips [23]-[27]. In all these drawing methods, probe tips are brought in contact with the dispensed polymer solution droplet on a substrate. The probe is then retracted, thus forming a liquid bridge between the probe tip and the dispensed polymer solution. This bridge increases in length and reduces in diameter and solidifies due to solvent evaporation.

This paper uses the drawing approach as a nanorobotic approach where a precise and repetitive control in the fiber geometry and form is aimed using proximal probes based drawing. As different from previous works such as [27], this paper proposes to also use glass pipettes for drawing fibers where the polymer solution comes out from the probe itself rather than having it on the substrate in advance. This novelty improves the initial droplet size down to tens of microns and also makes the drawing process much more reliable, repeatable, and longer since the solvent evaporation time problem is removed due to continuous supply/pumping of the solution through the pipette. Moreover, this is the first work showing the formation of microscale springs by probe based drawing techniques and showing preliminary simulation results for designing the nanorobotic drawing method parameters theoretically. Using systematic methods by modeling and proposing repeatable robotic drawing strategies, 1-D, 2-D, and 3-D custom polymer fibers with diameters down to few hundreds of nanometer are fabricated.

The paper is organized as follows. In Section II, fiber drawing strategy using different proximal probes is explained. Section III models the role of automation parameters such as speed of drawing and the velocity profile on the fiber drawing process using nonlinear viscoelastic finite element methods. In Section IV, a comparison of the experimental results obtained by using various proximal probes and polymer dispensing strategies is presented. In Section V, a methodology for the characterization of fibers drawn using AFM and Nanoindenter

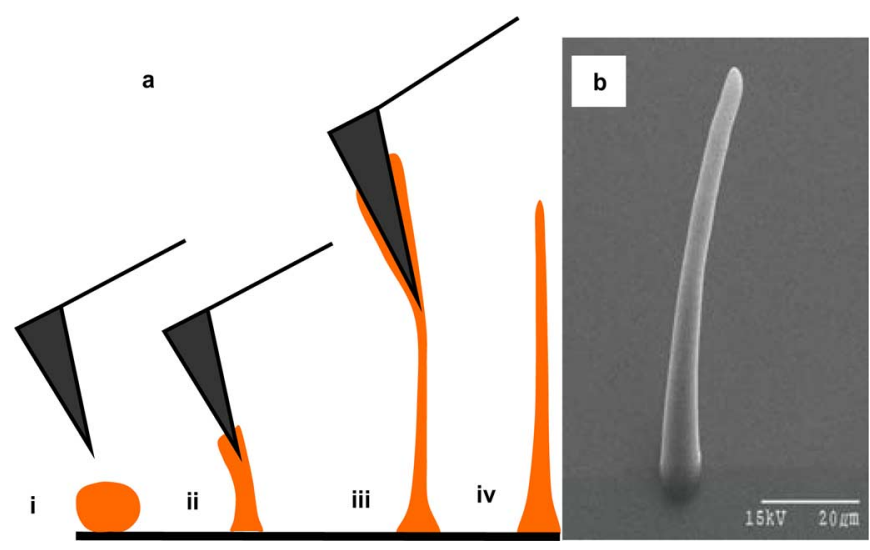

Fig. 3. (a) AFM/STM probe based fiber drawing strategy, i) probe tip approaches solution droplet, ii) liquid polymer bridge develops, iii) probe retracted forming fiber by solvent evaporation, iv) fiber detaches at the tip; (b) Scanning Electron Microscopy (SEM) image of a drawn vertical fiber.

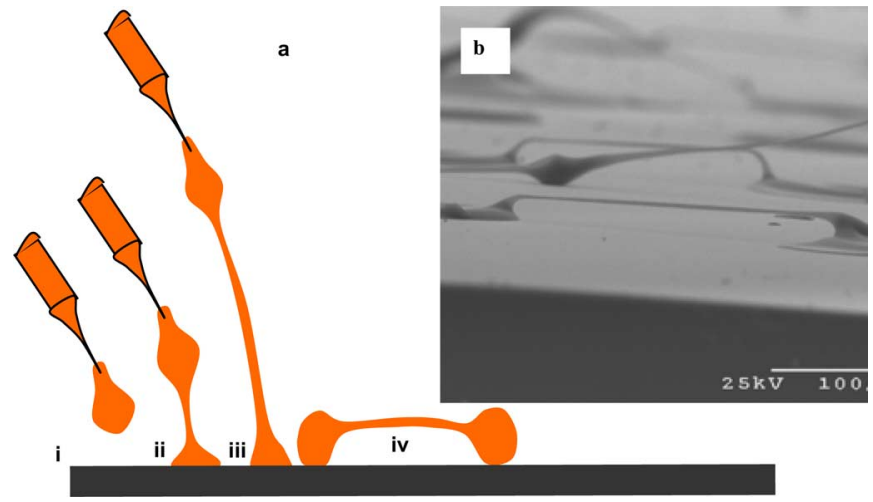

Fig. 4. (a) Micro-pipette probe fiber drawing strategy, i) probe/solution approach substrate, ii) formation of liquid bridge, iii) probe is retracted forming fiber by solvent evaporation, iv) probe/solution approach substrate again, fiber detaches from probe tip; (b) SEM image of a suspended fiber.

is proposed along with preliminary mechanical characterization data, and the various research issues that need to be addressed to mature this technology are reported in Section VI with conclusions in Section VII.

\section{Fiber Drawing Strategy}

In this paper, we propose fabricating fibers from solutions of thermoplastics dissolved in corresponding solvents. Dilute to semi-dilute polymer solutions are chosen for fiber formation as they are easier to form and require no specialized heating/cooling setups. Nanorobotic assisted solid (AFM or STM probes) or hollow (glass pipettes) micro/nanoprobes are combined with an appropriate polymer solution dispensing strategy for fiber drawing. Solidification of the fiber occurs due to solvent evaporation from the fiber surface during drawing, thus forming an amorphous polymer micro/nanofiber.

Drawing a fiber in both techniques (proximal probes and pipette delivery) differs only in the dispensing of the polymer solution as shown in Figs. 3 and 4. In the case of proximal probes, the AFM/STM tip is lowered until it makes contact with the already deposited polymer solution droplet on the substrate and the probe tip is retracted. The resulting liquid polymer bridge is then drawn along a predefined trajectory. In the case of the pipette delivery system, the probe tip with the ejected/pumped precalibrated volume of polymer solution 
is brought into contact with the substrate and is, thereafter, retracted, thus forming a liquid polymer bridge. As the tips (both cases) are retracted and moved along a predetermined 3-D trajectory, the liquid polymer bridge reduces in cross section, solidifies due to solvent evaporation and increases in length until it either finally breaks from the tip (Fig. 3) or is subsequently brought into contact with the substrate, thus forming a suspended fiber (Fig. 4). The ease of making fibers and the flexibility of 3-D shaping of polymeric material is clearly evident by using the micro-pipette delivery systems. The key problem associated with the AFM/STM probe system is the deposition of a small amount of a viscous polymer droplet on the substrate. Due to the high volatility of the solvent and high surface area to volume ratio at reduced length scales, there is a very small window of opportunity within which the fiber can be drawn. This is further complicated by the automation problems of locating the deposited droplet on the substrate and tracking the surface of droplet due to solvent evaporation.

\section{MODELING DRAWING OF A FIBER}

Simulations of fiber drawing provide us with the essential tools in exploring the material behavior under different fiber drawing parameters. In this section we present preliminary finite element analysis (FEA) simulation results of forming a 1-D fiber as a function of speed magnitude and profile.

Polymer solutions can be considered as a network of polymer chain entanglements in a solvent. These polymer chains align themselves in the strained direction which effectively decreases the number of entanglements relative to the unstrained state. At high strain rates significant deviations result from the linear viscoelastic regime due to the fact that more chains are displaced from their equilibrium conformations.

In this paper, the focus is to investigate the effects of automation parameters such as speed and the type of velocity profile on the fiber drawing process. The high deformation extensional flow experienced in fiber formation is not shear free and analytical solutions are complex. The simulations presented in this section are carried out in the commercially available software POLYFLOW which uses nonlinear constitutive equations simulating fiber deformation. This paper is important as the details of the key parameters in fiber formation are not well understood, and it is as yet unclear if a single control strategy and a set of key parameters will result in uniform fibers at reduced lengthscales for different polymer blends.

The mathematical modeling of flow, as described by the theory of continuum mechanics, is based on conservation equations and constitutive models. The equations are derived from the principles of conservation of mass, linear momentum, and energy. Constitutive models are material dependent and describe the stresses in the fluid as a function of the deformation history.

1) Model Verification: The dynamic behavior of extensional viscoelastic flows of polymers with solvents is typically modeled for validation against experimental results from Filament Stretching Devices (FSD) [28]. In the FSD, a cylindrical liquid column is first generated between two concentric circular plates and is then elongated by pulling one or both of the end-plate fixtures at an exponentially increasing rate. Due to the presence of deformable free surface and rigid end plates (no-slip
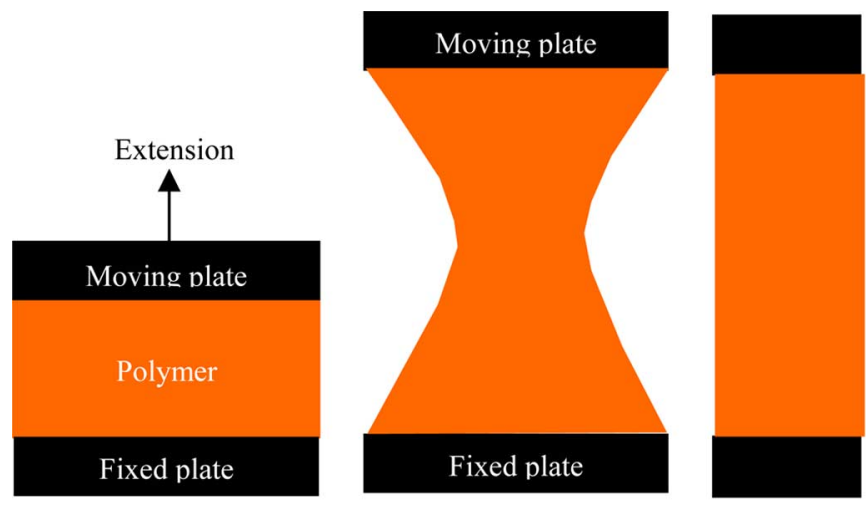

(a)

(b)

(c)

Fig. 5. Geometry for validation. (a) $t=0$; (b) $t>0$, FSD; (c) $t>0$, RDD.

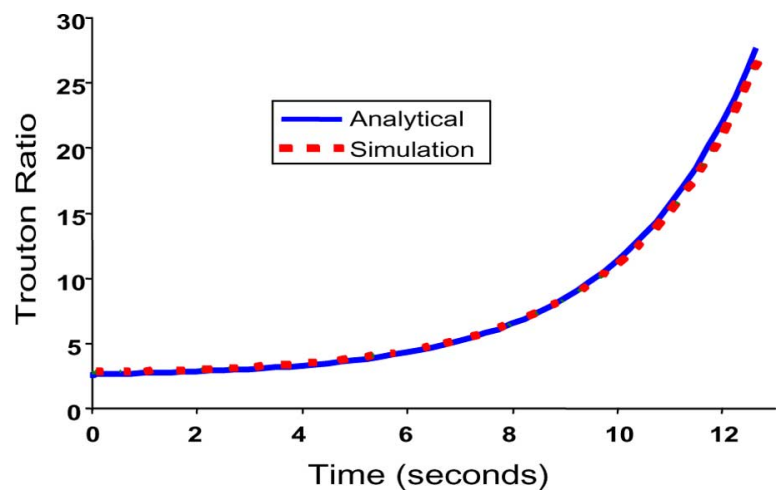

Fig. 6. Model verification: comparison of analytical and simulation results.

boundary condition), the elongation is not shear free, and the extension rate is spatially and temporally nonhomogeneous. Uniaxial flows are obtained by imposing a full-slip boundary condition at the rigid end plates in a Reducing Diameter Device (RDD). At time $t=0$ [Fig. 5(a)], the liquid bridge is elongated [Fig. 5(b)] from the moving end plate by imposing an exponential velocity, while the fixed end plate is kept stationary. At the same time, the radius of both end plates is simultaneously reduced exponentially, thus ensuring a full slip condition at both ends. This leads to a precise uniaxial elongation of the cylindrical fluid element [Fig. 5(c)]. The analytical solution for the extensional viscosity using the Oldroyd-B constitutive model [29] is

$$
\begin{aligned}
\eta_{E}=3 \eta_{S}+\frac{2 \eta_{P}}{1-2 \lambda_{1} \dot{\varepsilon}_{0}} & {\left[1-e^{-\left(1-2 \lambda_{1} \dot{\varepsilon}_{0}\right) t / \lambda_{1}}\right] } \\
+ & \frac{\eta_{P}}{1+\lambda_{1} \dot{\varepsilon}_{0}}\left[1-e^{-\left(1-\lambda_{1} \dot{\varepsilon}_{0}\right) t / \lambda_{1}}\right]
\end{aligned}
$$

where $\eta_{E}$ is the extensional viscosity, $\eta_{S}$ is the solvent viscosity, $\eta_{P}$ is the polymer viscosity, $\lambda_{1}$ is the relaxation time, and $\dot{\varepsilon}_{O}$ is the extension rate. Our results comparing simulations with analytical predictions are shown in Fig. 6, depicting an excellent agreement between theory and simulation and validate the choice of the constitutive model.

2) Fiber Drawing Simulations: Automating the fiber drawing process requires a detailed understanding of the key parameters involved in fabricating fibers at the submicron scale. Drawing fibers using traditional melt-spinning techniques have been studied in detail and there is extensive literature available. In the proposed approach the amorphous polymer fiber is formed at room temperature as opposed to the melt-spinning 


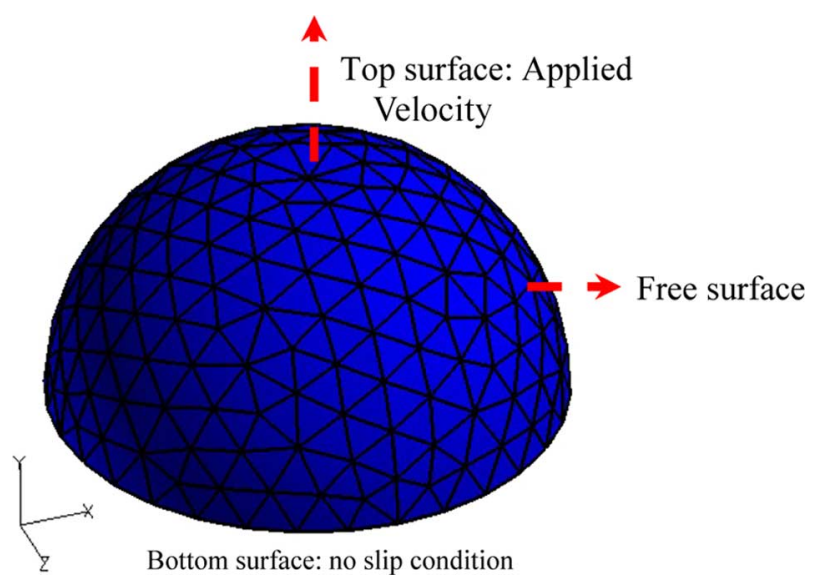

Fig. 7. Geometry and boundary conditions used in simulation.

process which can possess a certain degree of crystallinity. The dynamics of fiber drawing can be classified as dependent upon material properties, such as polymer-solvent viscosities, relaxation times and automation parameters such as speed of pulling, the type of velocity profile, contact conditions, probe tip diameters and polymer-solvent mass flow rates. The goal of this paper is to explore the effects of key automation parameters, namely the speed of drawing and choice of velocity profile on the fiber drawn.

a) Simulation domain: The geometry and boundary conditions are shown in Fig. 7. The simulation is initiated from the stage when the polymer has made contact with the substrate and the probe tip is retracted (Fig. 4). For a moderately hydrophobic surface, such as the silicon used in this study, the contact conditions at the bottom surface of the droplet (assumed to be $10 \mu \mathrm{m}$ in diameter) are assumed to have a negligible effect on the fiber being pulled at the top. Due to the stretching of the droplet, the simulation takes into account the free surface evolution and re-meshes the domain of the simulation at each time step.

PMMA material properties (Microchem Inc., USA) used in the simulation are as follows:

- Density $=1.113 \mathrm{~g} / \mathrm{cm}^{3}$;

- Viscosity of solution $=500$ centi-poise;

- Relaxation time $=1 \mathrm{~s}$;

- Chlorobenzene viscosity $=0.8$ centi-poise.

The investigation was carried out for only a quarter of the domain as the geometry is asymmetric, and 3-D tetrahedron elements (1232 elements) were used to mesh the quarter domain.

b) Constitutive model: The Giesekus constitutive model accurately predicts the nonlinear deformation behavior of concentrated polymer solutions in organic solvents [30] based on the concept of deformation-dependent mobility. In the singlemode formulation of the model, the solvent contribution $\tau_{s}$ and the polymeric contribution $\tau_{p}$ to the extra stress are defined as [29], [31]

$$
\begin{aligned}
\tau= & \tau_{s}+\tau_{p} \\
\tau_{s}= & \eta_{s}\left(\nabla \nu+(\nabla \nu)^{T}\right) \text { and } \tau_{p} \\
& +\lambda\left(\frac{\partial \tau_{p}}{\partial t}+\nu \cdot \nabla \tau_{p}-(\nabla \nu)^{T} \cdot \tau_{p}-\tau_{p} \cdot \nabla \nu\right) \\
& +\frac{\alpha \lambda}{\eta_{p}}\left\{\tau_{p} \cdot \tau_{p}\right\}=\eta_{p}\left(\nabla \nu+(\nabla \nu)^{T}\right)
\end{aligned}
$$

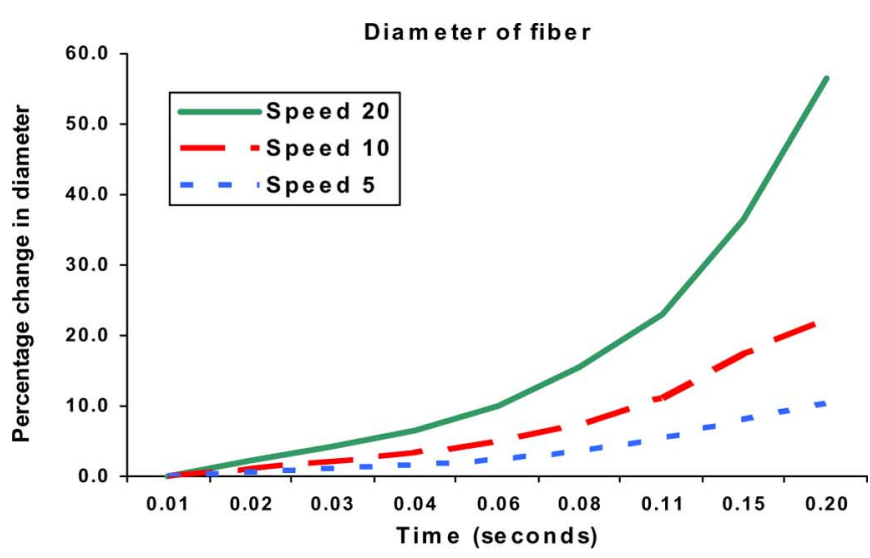

(a)

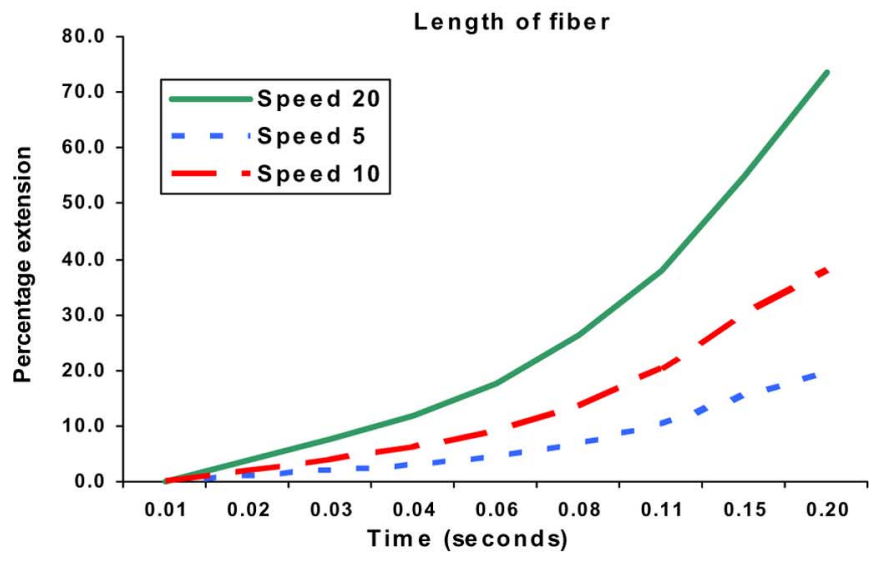

(b)

Fig. 8. Effect of varying velocity on (a) fiber diameter, (b) length of fiber.

where $\eta_{s}$ and $\eta_{p}$ are the solvent and polymer contributions to the viscosity, respectively, $\alpha$ is a dimensionless number associated with the anisotropic effects, and $\lambda$ is the relaxation time. For physically meaningful results with the Giesekus model, it is required that $0 \leq \alpha \leq 0.5$. For this paper, a value of $\alpha$ to be 0.25 was chosen. In the limit $\alpha \rightarrow 0$, the single mode Giesekus model reduces to the quasi-linear Oldroyd-B model and higher values of $\alpha$ lead to shear thinning in the material.

c) Simulation parameters: This study was undertaken to establish the fiber formation capabilities of viscoelastic solutions from the automation perspective. It is anticipated that different materials will have different fiber forming capabilities, but the effects of automation parameters will exhibit similar behavior in all variations. The speed of drawing and the velocity profile are of key interest. The inner diameter of the probe tip is an important parameter that is usually not considered due to the fact that with the decrease in diameter a higher pressure is required to pump the same viscosity polymer solution or, alternatively, a less viscous polymer solution is used at the same pressure. Automation parameters will apply to and show similar trends in the evolution of the fiber for either case. The effect of speed on the diameter and the length of the fiber formed is shown in Fig. 8(a) and (b). The speed was varied from 5 to $20 \mu \mathrm{m} / \mathrm{s}$ and the simulation run time was set to 0.2 seconds. The length of the fiber approximately doubles with the change in the speed whereas the diameter of the fiber decreases in a nonlinear fashion for the range of parameters investigated. At low speeds 


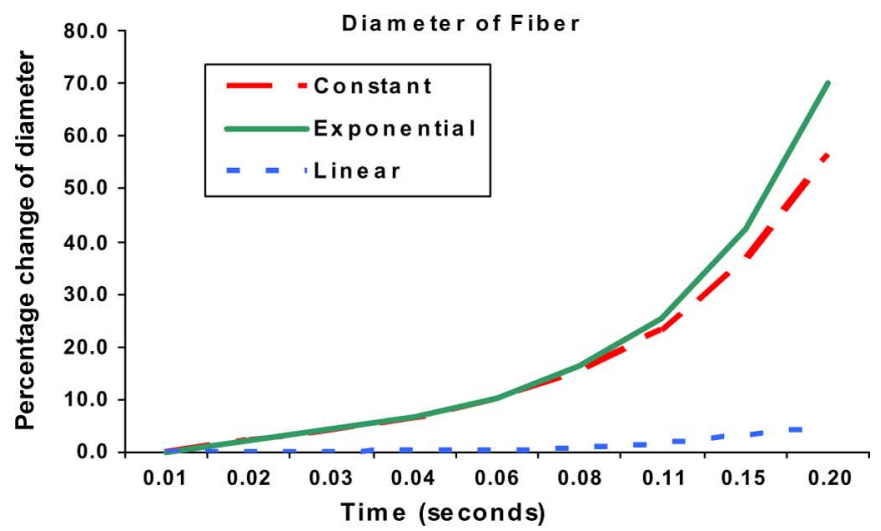

(a)

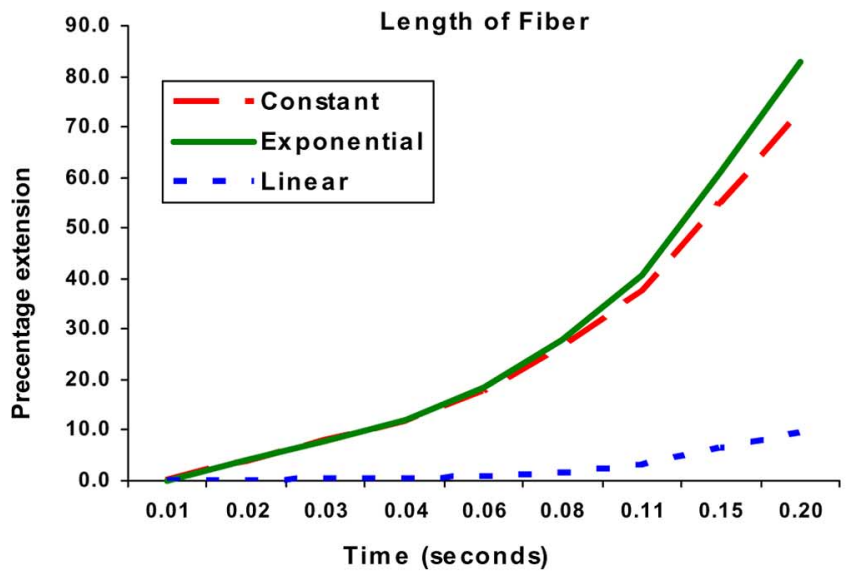

(b)

Fig. 9. Effect of varying velocity profile on (a) fiber diameter, (b) length of fiber.

the viscous effects are dominant and the elastic effects are easily visible at higher velocities leading to higher deformations. This particular feature is very important from the automation standpoint as higher deformations lead to strain hardening effects in the polymer which will lead to higher stresses or forces on the probe tip. This may allow for developing real time force measurement techniques on the probe tip in the future.

The second automation parameter simulated is the type of velocity profile (Fig. 9) with which the probe tip is retracted. A base velocity of $20 \mu \mathrm{m} / \mathrm{s}$ was chosen along with the evolution velocity profile. For uniaxial stretching flows, a constant strain rate is obtained in an RDD by applying an exponentially increasing velocity profile. Due to the imposed boundary conditions in this study, it is not possible to obtain a constant strain rate due to the presence of shear effects. Three realistic velocity profiles, which can be generated by the controller moving the probe tip, were chosen: exponential, linear and constant velocity. The highest deformation was observed for the exponential velocity profile. The linear velocity exhibits mostly viscous effects and minimal deformations. Using an exponential velocity profile instead of a regular constant velocity profile yields an approximate 10\% increase in the fiber length and an approximate $14 \%$ reduction in the fiber diameter.

However, it should be noted that in these simulations the solvent evaporation rate has not been included due to the limitations of the software. It is expected that the solvent evaporation rate will be an important factor in fiber formation because
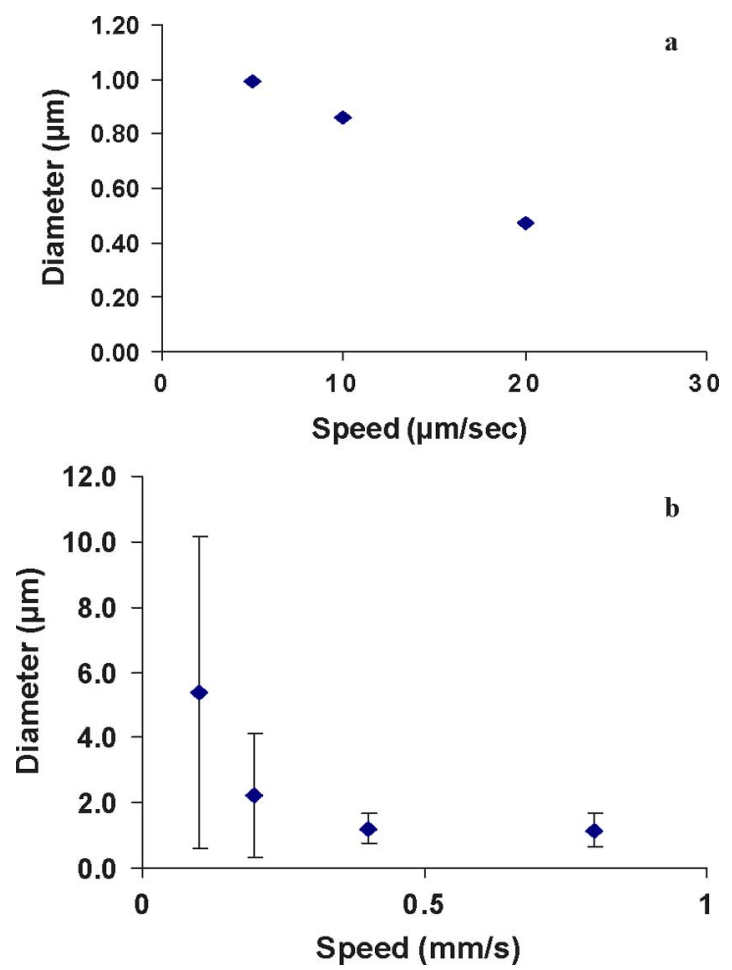

Fig. 10. Diameter of drawn fiber as a function of speed; (a) simulations and (b) experiments.

the solidification that results from solvent evaporation involves irreversible changes in structural and macroscopic characteristics of the material [32]. Evaporation of the solvent from the polymer solution increases the viscosity and the relaxation time and, thus, has a direct effect on the thinning characteristics of the fiber.

Simulation results indicate that higher drawing speeds lead to thinner and longer fibers thus enhancing the solvent evaporation rate from the reduced cross sections. This may have undesirable effects on the fiber formation capabilities and lead to early breaking of the fibers from the probe tip. The effect of solvent evaporation can be minimized by using low vapor pressure solvents and/or by performing the experiments in controlled and/or solvent vapor rich environments.

d) Comparison of simulation and experiments: In Fig. 10, we present the results of experiments carried out to determine the effect of automation parameter "speed" on the diameter of the drawn fiber. The difference in magnitude of the applied speed is due to the limitation imposed by the simulation tool to model the solvent evaporation rate. At very low speeds, sufficient solvent evaporation occurs, thus leading to the formation of a skin on the fiber during the fiber process which leads to fibers having uneven structures. Thus, experimentally, the best speed values were determined and, as in simulations, the speed was doubled to determine the effect of speed on the diameter of the fiber. The experimental study was carried over approximately 50 fibers for each speed. Both simulation and experimental data suggest a rapid decrease in the fiber diameter with an increase in speed. However beyond a certain range of speed, the final diameter does not vary. This finite range of speeds is a variable which requires detailed investigation; however, for the present study, the simulations provide an easy method to investigate the design space of drawing fibers. 


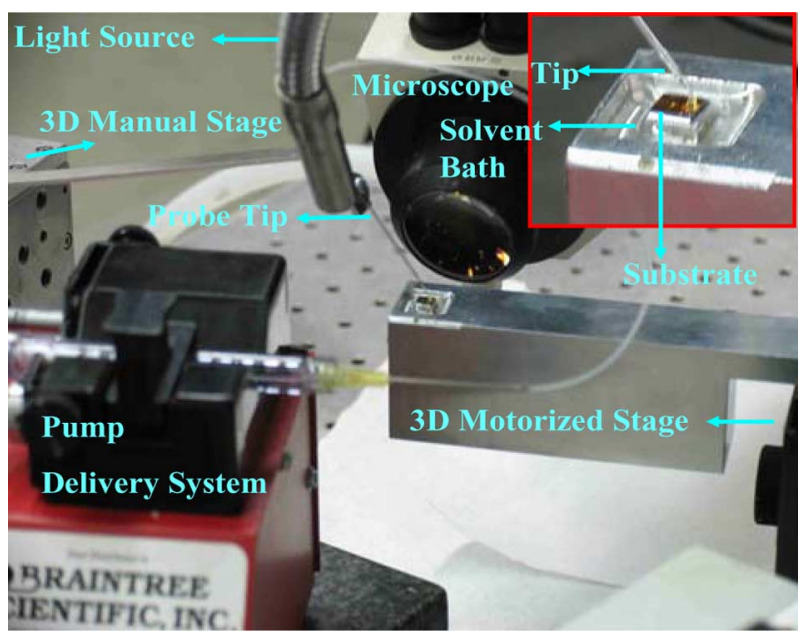

Fig. 11. Photograph of the experimental setup for glass micro-pipette based drawing. Inset shows the zoomed view of substrate and probe tip in an island of solvent bath.

\section{EXPERIMENTS}

For pulling experiments, poly (methyl methacrylate) (PMMA) thermoplastic was chosen as it is a widely used and characterized polymer which has superior fiber forming and optical properties. It is easily available in a range of molecular weights with narrow polydispersities. PMMA, with a molecular weight of $950 \mathrm{~K}$ dissolved in chlorobenzene $(9 \%$ by weight, Microchem Inc.), was used in this study.

\section{A. Experimental Setup}

1) AFM/STM Probe Based Drawing: For experiments, a Park Scientific Instruments Autoprobe M5 with Park Scientific Instruments noncontact silicon Ultralevers (stiffness $\geq 20 \mathrm{~N} / \mathrm{m}$ ) were used. Low stiffness cantilevers were not suitable for pulling fibers from viscous mediums as the elastic recoil of the fiber being pulled would break the tip. The high stiffness probes, on the other hand, were prone to being completely immersed in the polymer droplets. Moreover, tungsten STM probes (Veeco Inc.) with sharp tips down to 50-100 nm diameter and very high stiffness were used under an optical microscope for their contact detection with the substrate and the polymer solution droplet.

2) Glass Micro-Pipette Probe Based Drawing: A custom setup (Fig. 11) was built in which a silicon or polydimethylsiloxane (PDMS) substrate was vacuum mounted on a XYZ high precision motorized positioner (Newport, V20X) with $0.1 \mu \mathrm{m}$ precision and $25-\mathrm{mm}$ range with a built in solvent reservoir. The fixed probe was mounted on a manually adjustable micro-positioner for fine alignment and calibration. The polymer solution was pumped into the probe tip via a flexible hosing, which was connected to a pump delivery system for pumping a calibrated amount of polymer solution. In our preliminary attempts, all the experiments were carried out with the aid of side-view optical feedback, as shown in Fig. 11. The probes with tip diameter around $500 \mathrm{~nm}$ were fabricated using a glass puller machine (P-97 Sutter Instruments) as shown in Fig. 12.

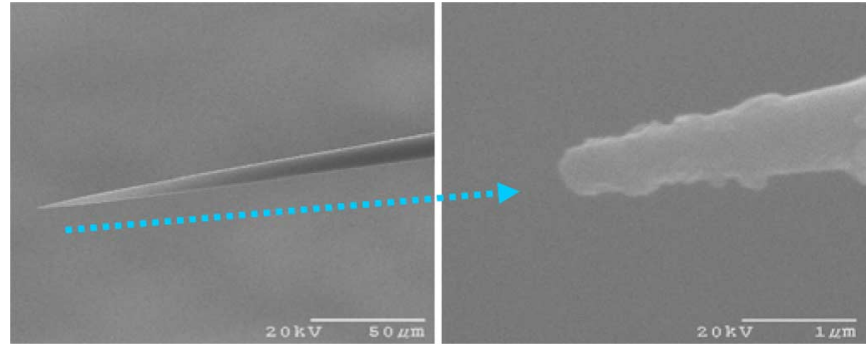

Fig. 12. SEM image of a typical micro-pipette used in experiments.

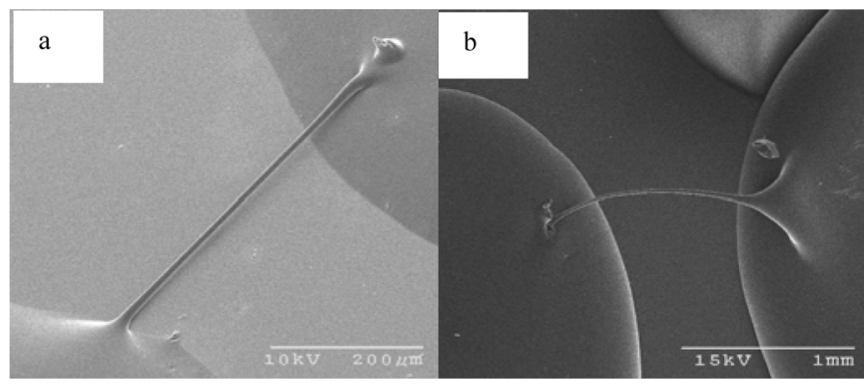

Fig. 13. SEM images of horizontal fiber s pulled using an AFM probe (a) on a silicon substrate (b) on a PDMS substrate exhibiting hydrophobicity.

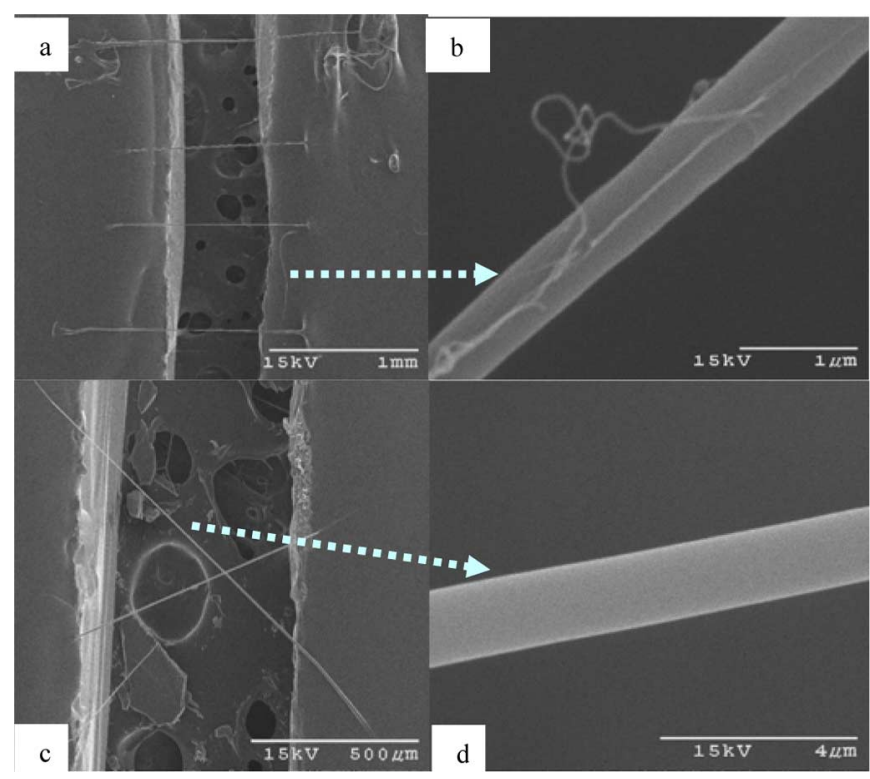

Fig. 14. SEM images of STM probe based drawing: (a) parallel fibers, (b) micro/nanofiber, (c) cross formation, (d) submicron fiber.

\section{B. Experimental Results}

1) AFM/STM Probe Based Drawing: Horizontal fibers (Fig. 13) were drawn by depositing two polymer droplets on the substrate and the probe was used to draw a fiber between them. Different arrangements of fibers were formed between two substrates as shown in Fig. 14. Moreover, microscale springs (Fig. 15) were formed by a fast drawing of the formed polymer fiber and then breaking the fiber from the tip instantaneously. We hypothesize that the broken fiber self-organizes into a spiral structure after the stretched fiber is broken. These springs could be used as mechanical components of MEMS structures and miniature robots. Analysis and experimentation of repeatable and controlled pulling of micro-springs is our future work. 


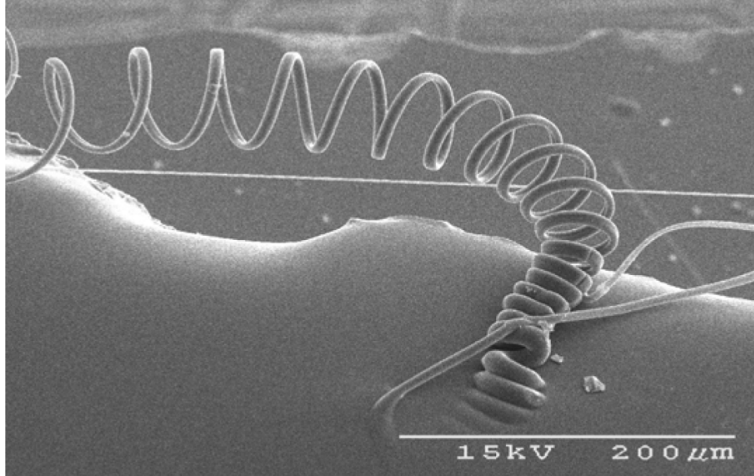

Fig. 15. SEM image of a polymeric spring drawn using an STM probe.

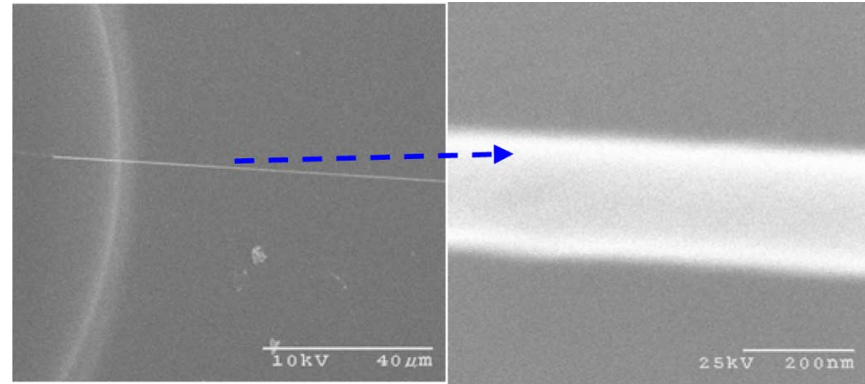

Fig. 16. SEM images of a sample suspended polymer fiber with approximate diameter of $200 \mathrm{~nm}$.

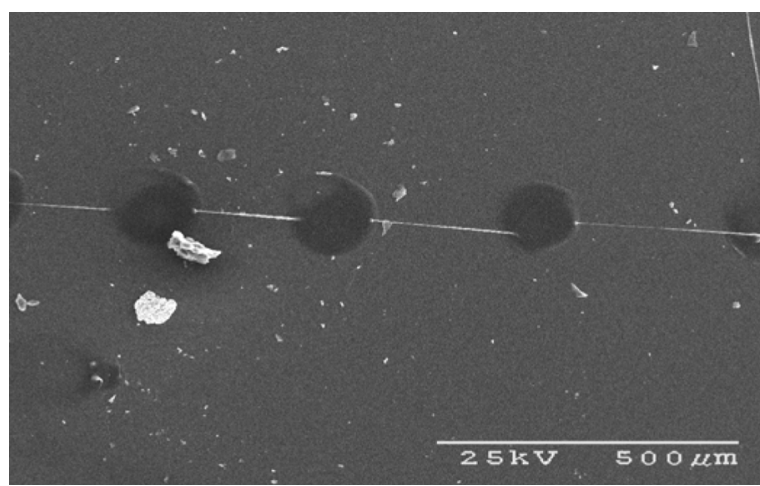

Fig. 17. Stitching operation exhibiting the repeatability.

\section{2) Micro-Pipette Based Drawing:}

a) Experiments: Very high aspect ratio (length to diameter ratio) horizontal fibers with diameters less than $200 \mathrm{~nm}$ have been fabricated using this approach (Fig. 16). One key advantage to this method is the repeatability of stitching a surface (Fig. 17) as it provides the ability for a continuous deposition and shaping of a calibrated polymer material in predefined spatial coordinates. Next, highly oriented polymer fiber networks, with a mix of polymer fiber diameters within the same network, are shown in Fig. 18.

b) Effect of speed on drawing fibers: Simulation predictions showed a rapid decrease in the fiber diameter with the increase in the speed of drawing. Fig. 19 shows the effect of drawing speed on the final diameter of the fiber. Due to the limitation of the simulation software to model the solvent evaporation, experiments were developed to isolate the effects of solvent evaporation by using the same setup, boundary and operating conditions and varying only the speed. Even though the solvent evaporation effects have been minimized in the

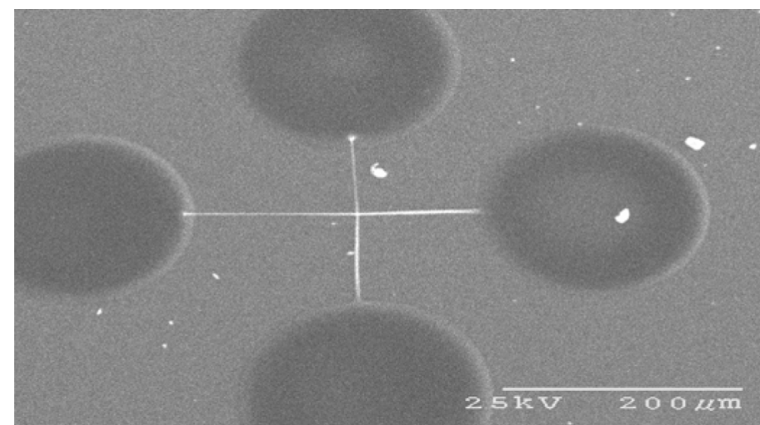

Fig. 18. Crossed network of polymer fibers.

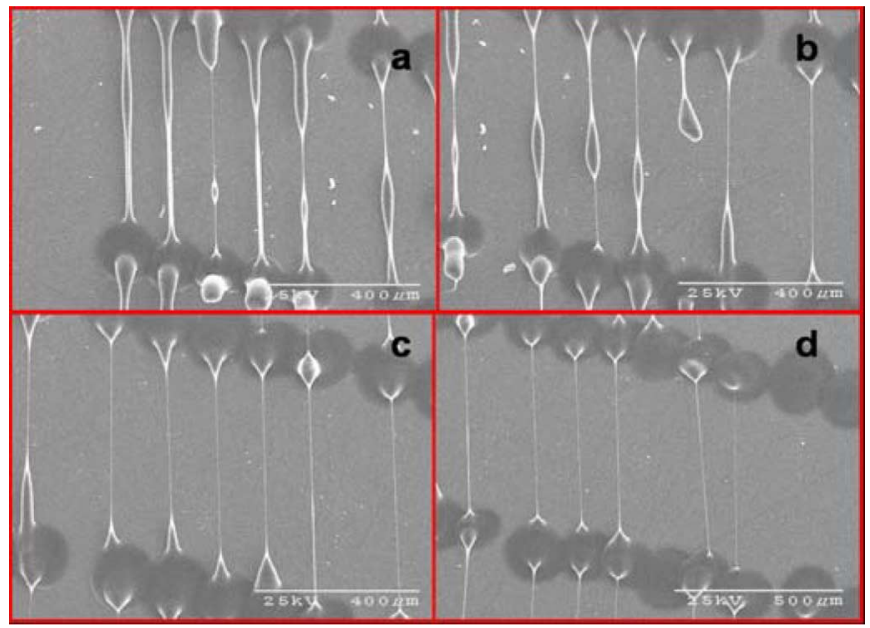

Fig. 19. SEM images of experiments showing the effect of increasing speed on the fiber quality and diameter, (a) $0.1 \mathrm{~mm} / \mathrm{s}$, (b) $0.2 \mathrm{~mm} / \mathrm{s}$, (c) $0.4 \mathrm{~mm} / \mathrm{s}$, and (d) $0.8 \mathrm{~mm} / \mathrm{s}$.

experiments, but they still cannot be negated, thus, only the qualitative effects of speed on drawing fibers are discussed. At low speeds [Fig. 19(a)], the fibers formed are big and lack overall good quality, besides having discontinuous regimes. Doubling the speed [Fig. 19(b)] improves the quality of the fibers marginally, besides increasing the uniformity of fibers. Doubling the speed again [Fig. 19(c)] shows good quality and uniformity of fibers drawn which are distinctively smaller than the earlier two sets. The fibers drawn still have irregularities in structure near the start and end points. Doubling the speed once again [Fig. 19(d)] shows a marked improvement in the structures and uniformity of fibers. Experiments were carried out beyond these speeds, but the effect of speed was not found to have a significant change in the fiber diameter. The decrease in the diameter of the fiber as a function of drawing speed is presented in Fig. 10.

Often, fibers need to be deposited on geometries where it is, if not possible but very difficult to deposit droplets and pull fibers using the AFM/STM probe based approach. In Fig. 20 we show the ease with which fibers can be deposited on two such geometries: right angled surfaces [Fig. 20(a)] and curved surfaces [Fig. 20(b)], using the pipette based approach, thus exhibiting the 3-D capabilities of this approach.

It is clear from the images that the micro-pipette probe strategy is superior such that it allows for the fabrication of fibers with diameters in the sub-200 $\mathrm{nm}$ range, along with the repeatability, customized deposition, and the shaping of 

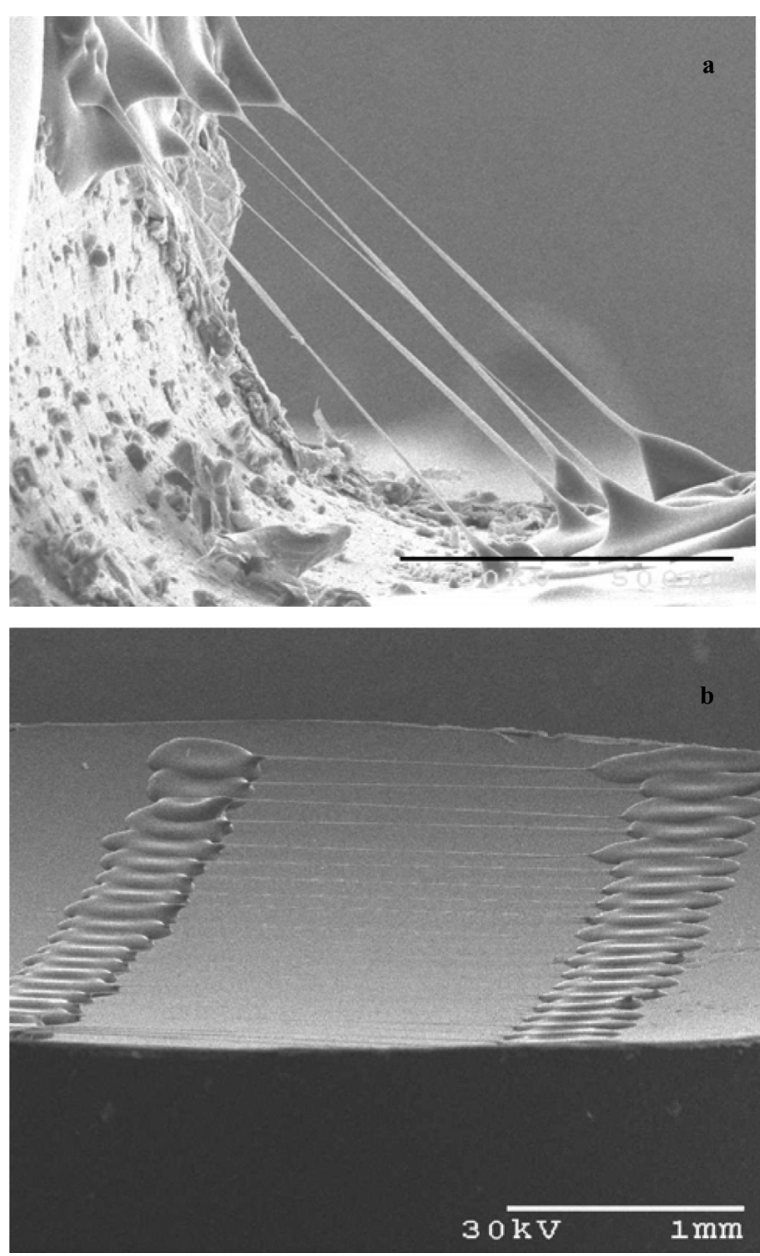

Fig. 20. SEM images of fibers deposited on (a) right-angled surfaces (scale bar shown is $500 \mu \mathrm{m}$ ), (b) curved surfaces.

polymeric material. A distinct advantage of this technique over other probe types is the elimination of polymer solution deposition on the substrate prior to drawing, which offers a small timeframe to draw fibers. Various architectures of polymeric fibers can now be realized in 1, 2, and 3 dimensions as there is no limitation of the deposition of the second droplet as outlined in the AFM/STM probe based approach in this paper and in [27]. This approach, as opposed to the AFM/STM method, now offers active control on the processing parameters, which has a direct bearing on the fiber fabrication capabilities. This enables on a repeated basis to forms fibers of uniform diameter and the desired aspect ratio. These features vastly increase the number of applications to which this technique can be applied for realizing novel technologies.

\section{Mechanical Characterization OF Fibers}

Since the mechanical properties of the micro-pipettes based pulled micro/nanofibers could vary from the bulk polymer material significantly, a preliminary mechanical characterization scheme of suspended fibers using an AFM and a Nanoindenter setup is proposed in this paper. AFM and Nanoindenter have been used extensively in the study of material properties and topology, but with the proposed method of fabricating free

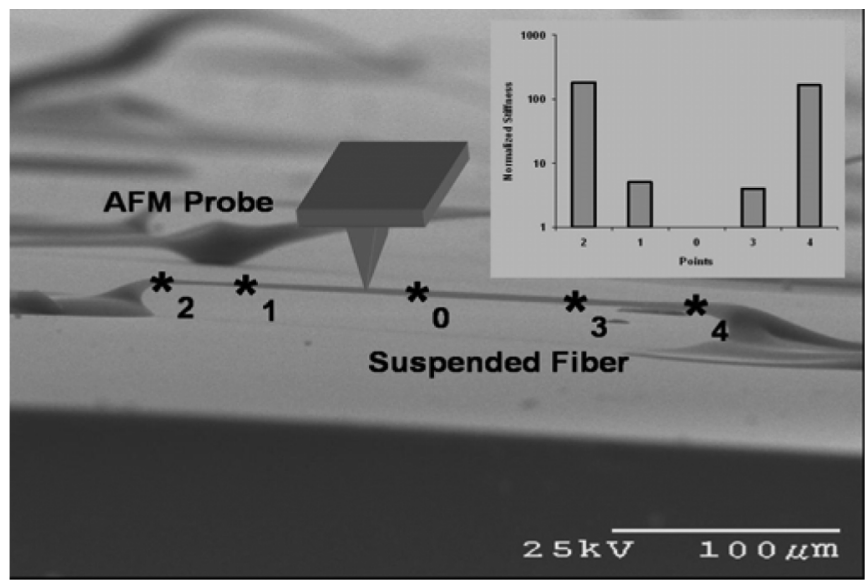

Fig. 21. Schematic of AFM setup (not to scale) and the variation in fiber stiffness along the span of the fiber at the five chosen points for experiments performed on 16 fibers. Inset shows the increase in stiffness normalized with respect to point 0 .

standing high aspect ratio polymeric fibers; these tools provide new opportunities for probing the mechanical properties of fibers. AFM (normal force) and nanoindentation (lateral force) techniques have been explored to determine the stiffness, elastic modulus, and the forces required to break a fiber.

1) Fiber Stiffness Measurements: AFM force-distance curves are used to measure the fiber flexural stiffness. A high stiffness (Nanosensors Inc., $42 \mathrm{~N} / \mathrm{m}$ ) cantilever was used in the experiments at five different locations as shown in Fig. 21. In order to perform these measurements, high aspect ratio PMMA fibers with bigger diameters and larger separations from the substrate were fabricated. The deflection in the force-distance data is a combination of probe and fiber deflection. It is assumed that the major contribution to this deflection is from the fiber due to the choice of high stiffness probe. Due to geometrical difficulties in aligning the tip end with the top of the fiber, it was decided to make contact with the leading edge of the cantilever and not the tip. This avoided the coupling of extraneous attractive forces from the cantilever geometry. The cantilever end was optically aligned with the fiber and contact was established. The measurement points were selected by looking at the fiber in the AFM setup under a microscope, and the separation distance between them was set manually. Points 1 and 3 were chosen sufficiently away from point 0 and the corresponding AFM force-distance plots are shown in Fig. 22 indicating a sharp increase in stiffness. Points 2 and 4 were chosen to be the sharp transition of the fiber towards the substrate. The inset in Fig. 21 shows the variation of the fiber stiffness measurements at the chosen points for sixteen fibers. In the experiments, attempts were made to minimize the variance in the spatial location of the points. However this variance does not introduce any error in the measurement technique due to the low stiffness of the fiber in the extended middle region of the fiber and the rapid increase in the stiffness of the fiber at the narrower lengths near the ends. Fig. 23 shows the rapid increase of the fiber stiffness from points 1 and 3 towards points 2 and 4, respectively. This three hundredfold increase at the end of the fiber demonstrates that the fiber ends are rigid and do not rotate for the ranges of 

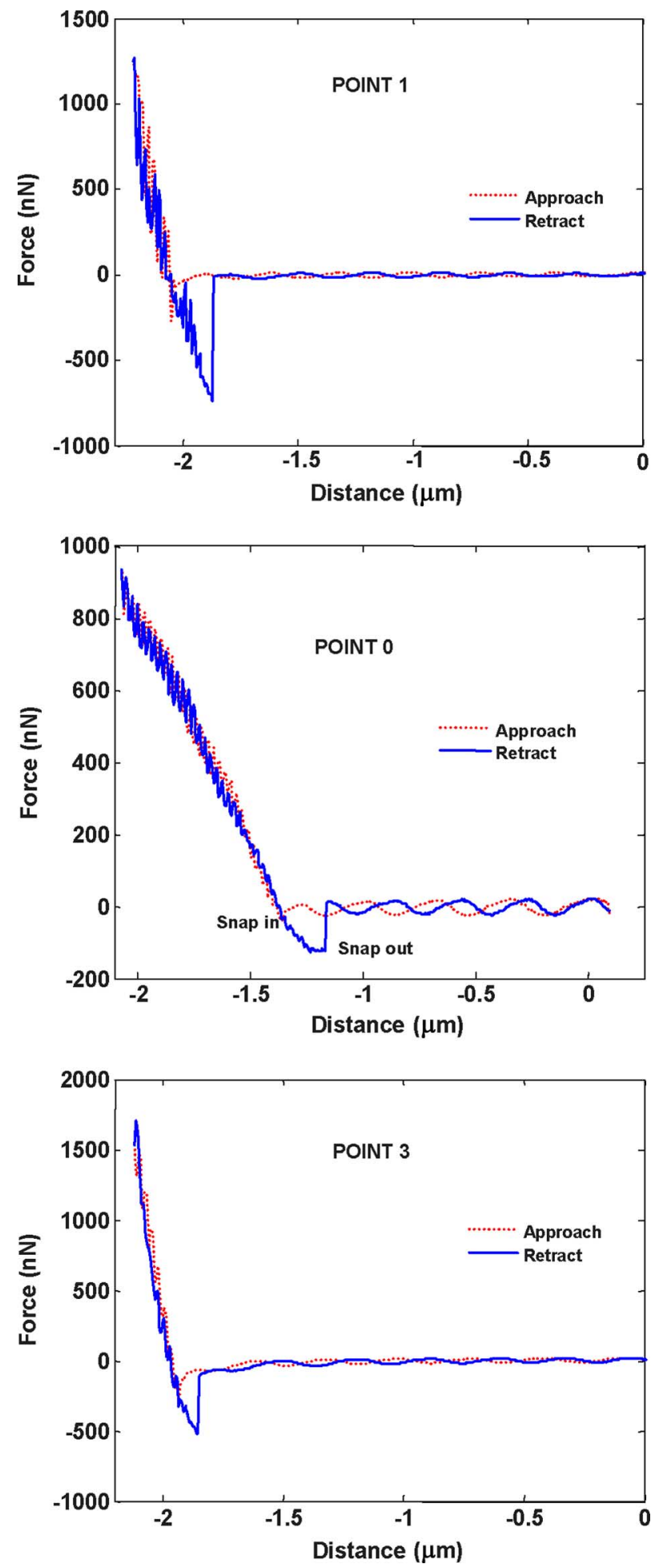

Fig. 22. Typical force-distance curves obtained at points 0,1 and 3 .

force-distance measurements performed at the mid point of the fiber.

The resultant stiffness values using springs- in-series solution at the three points for the two fibers characterized are tabulated in Table I. As expected, point 0 being at the center would have the maximum deflection and the least stiffness, with maximum stiffness being where the fiber geometry makes a sharp transition as shown in Fig. 23.

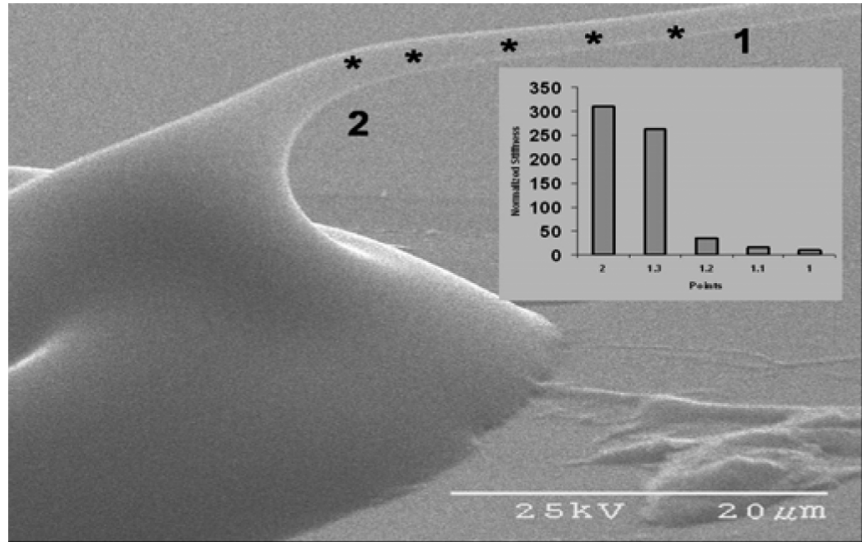

Fig. 23. Variation in fiber stiffness between points 2 and 1.

TABLE I

STIFFNESS VALUES (N/m)

\begin{tabular}{|c|c|c|c|}
\hline Fiber & Point 1 & Point 0 & Point 3 \\
\hline 1 & 34.88 & 2.26 & 15.05 \\
\hline 2 & 22.68 & 7.81 & 22.58 \\
\hline
\end{tabular}

The classical three-point bending test can be employed in this case to determine the elastic modulus of the suspended fiber with the edges clamped. The modulus is related to the spring constant by

$$
E=\frac{4}{3} K \frac{L^{3}}{\pi d^{4}}
$$

where $K$ is the stiffness of the fiber at the middle point $\mathrm{B}, L$ is the total length of the fiber and $d$ is the diameter of the fiber. The fiber length and diameter are estimated approximately from Fig. 20 to be 200 and 5-6 $\mu \mathrm{m}$, respectively, which gives a modulus value of 5-12 GPa. This modulus is slightly higher than the typical bulk PMMA compressive modulus of 2.5-3.1 GPa. The increase in the modulus can be attributed to the aligned state of the molecules in the stretched fiber which imparts higher mechanical values.

2) Fracture Strength of Fibers: Nanoindentation technique, which is often applied for probing mechanical properties of polymer materials locally, can be used to determine the fracture strength of the suspended fiber as shown schematically in Fig. 24(a). A Berkovich tip is preloaded and moved in the transverse direction until it makes contact with the fiber. The lateral forces experienced by the probe begin to increase and the fiber starts to deform until it fractures, thus giving the force required to fracture the fiber. In our preliminary experiments using nano-indenter, fibers undergoing lateral extension have been fractured, as shown in Fig. 24(b) and (c). A plastically deformed fiber which could not be fractured due to the force limitations of the nanoindentor is shown in Fig. 24(d). Two suspended fibers were loaded in the lateral direction in the nanoindenting setup and the tip was optically aligned with the fiber. The tip was preloaded to $100 \mu \mathrm{N}$ and moved automatically in the lateral direction. The lateral forces required to break the fiber are shown in Fig. 25 and it shows bigger fibers requiring higher 


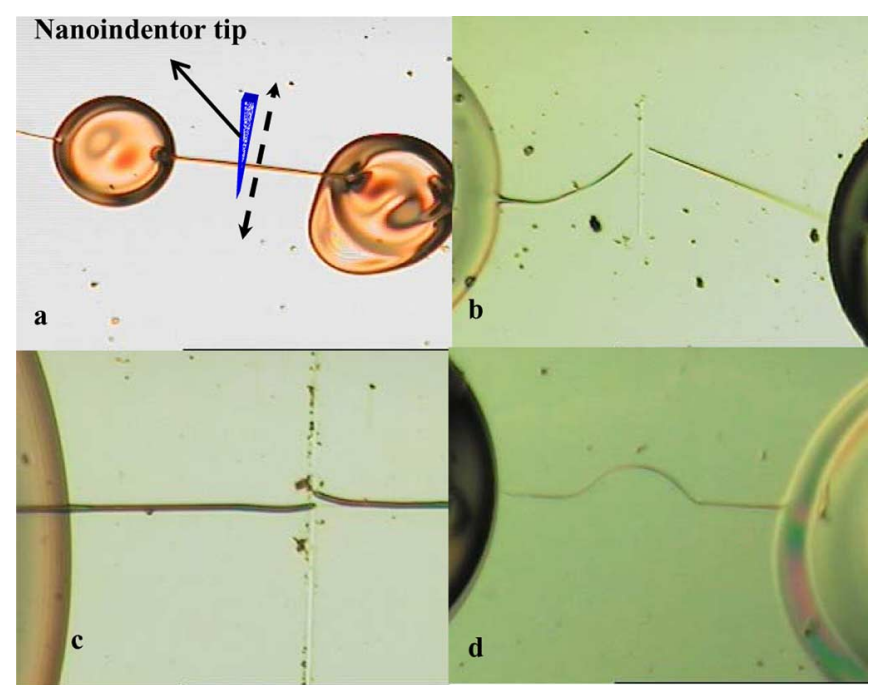

Fig. 24. Lateral force measurement (a) schematic of strategy, (b) and (c) optical image of fractured fibers, and (d) optical image of plastically deformed fiber.

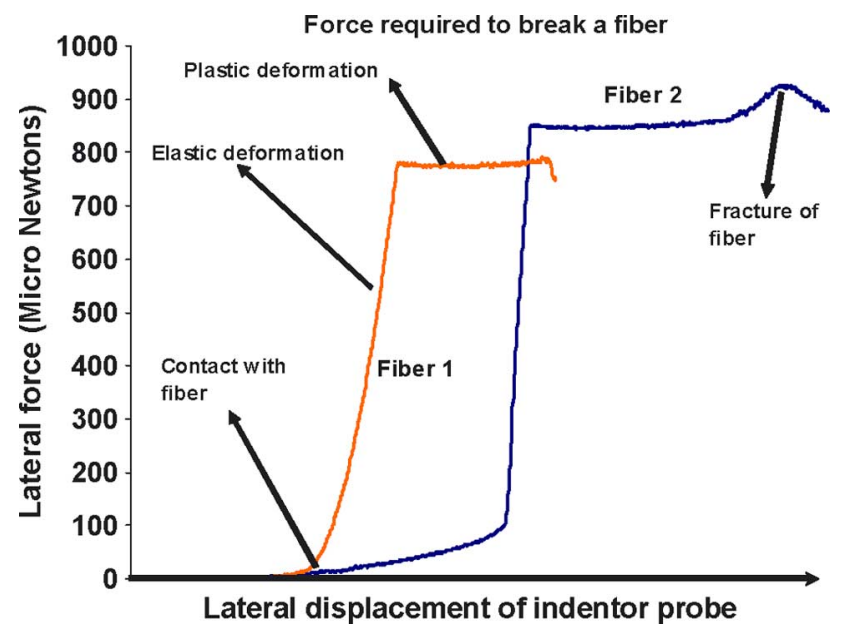

Fig. 25. Lateral force measurements using nanoindenter for two fibers exhibiting the typical viscoelastic behavior.

fracture forces. The lateral force increases as the tip makes contact with the fiber, which is followed by the elastic deformation. This is followed by the plastic deformation of the fiber at higher strains and finally, the fiber fractures upon which there is a decrease in the lateral force. The ultimate tensile strength of the fibers ranging in diameters from $700 \mathrm{~nm}$ to $1.5 \mu \mathrm{m}$ is found to be $324 \mathrm{MPa}-1.3 \mathrm{GPa}$. The values reported are higher than the bulk tensile strength values of PMMA (55-80 MPa) and can be attributed again to the aligned state of the polymer molecules in the uniaxially stretched fiber.

The proposed mechanical characterization of the PMMA polymer micro/nanofibers shows a deviation from the bulk properties and can be explained by the molecular network of the chains in the fiber. The molecular chains in the entangled random network undergoing extensional flows tend to be highly aligned in the fiber formation process. Thus, the suspended fibers are in stressed form, and, as the fiber dimensions decrease, it is expected to see a more pronounced deviation from the bulk properties. Existing mechanical techniques to characterize the mechanical behavior of fibers fabricated using the electrospinning process suffer from orientation of the fibers in the deposited woven mats along with the non homogenous fiber diameter uniformity [33], [34]. The proposed mechanical characterization techniques take on importance and relevance with the reduction in the length scales of the fibers formed specially when coupled with the proposed fiber forming technique.

\section{OPEN ISSUES}

The feasibility of controlled pulling of PMMA fibers down to few hundred nanometer diameters is demonstrated. However, there are many open challenges to mature and automize the pulling system for nanomanufacturing applications. Some major open issues are discussed below.

1) Tip-Substrate Distance Calibration: In order to automate this approach, it is very important to locate the surface of the substrate with respect to the tip. This is coupled with the problem that the polymer is being ejected on a continual basis and, thus, forms a droplet at the tip. This calibration can be accomplished by force feedback or electrical measurement by using a conductive tip and substrate.

2) Force Sensing on the Probe: Real time measurements of the forces experienced on the probe tip during the fiber drawing process can indicate the size of the fiber being pulled. This is crucial as the tip gets clogged or the polymer adheres to the outer surface due to solvent evaporation, thus leading to either bigger fibers or no fibers being formed. The envisioned system will allow real time force sensing at the tip, which will determine the preventive measures to be taken before the tip is rendered useless for pulling.

3) Calibration of Polymer Volume Deposited: Precise calibration of the polymer volume ejected per fiber fabricated would allow for minimal droplet size on the substrate. This would minimize the polymer wastage per fiber fabricated and allow this technique to fabricate fibers on even smaller structures such as MEMS elements.

4) Polymer Properties: A detailed experimental and analytical study needs to be undertaken to establish the effect of material properties, such as molecular weights and viscosities, along with the automation parameters, to optimize fiber fabrication for the same polymer in different blends.

5) Application Oriented Experiments: Conductive polymer blends such as polyanaline offer the potential to be drawn into thin wires which could serve as interconnects in MEMS and flexible substrate architectures. Polymers with superior fiber forming properties (Kevlar, polystyrene, polyethylene, etc.) can be explored as possible mechanical connecting elements in MEMS or can find applications in textile industry.

6) Characterization of Polymer Fibers: Mechanical and electrical characterization of polymer fibers as a function of density, diameter and aspect ratios would enable exploring the potential applications. Using the proposed mechanical characterization schemes, a detailed study undertaken to determine the mechanical properties of stand alone single fibers as a function of material and automation parameters will greatly extend the knowledge on molecular conformations in the fiber and the fiber drawing process.

7) Multiprobe Based Automation Schemes: Future networks of polymer fibers would entail fabricating multiple fibers, which 
is a very tedious fabrication procedure, if attempted sequentially. Design architectures of multiple probes, with each probe having individual force sensing, dispensing, and motion capabilities would allow for creation of such networks in parallel. With such capabilities it would then be possible to create sophisticated networks consisting of different fiber diameters, materials, geometrical spacing, and orientation of the different layers in the network.

\section{CONCLUSION}

In this paper, polymer fiber drawing strategies using different proximal probes have been explored and it has been demonstrated that the micro-pipette strategy has superior advantages over other proximal probe-based strategies. Suspended high aspect ratio PMMA fibers (diameter down to $200 \mathrm{~nm}$ and length longer than $200 \mu \mathrm{m}$ ) and networks of fibers have been successfully fabricated using the proposed approach. The nanorobotic assisted micro-pipette based technique provides a robust and repeatable approach to deposit polymer solutions in a customized three dimensional paradigm. The flexibility and ease of depositing polymer solutions as fibers provides unprecedented opportunities for maturing enabling technologies employing nanomanufacturing test beds.

Automation parameters have been studied and their effects on fiber formation have been investigated using nonlinear viscoelastic finite element simulations. Increasing the speed of drawing leads to smaller diameters has been demonstrated both experimentally and through simulations. Exponential velocity profile is shown to give smaller fiber diameters. A methodology for mechanically characterizing the fibers has been proposed and preliminary mechanical characterization indicates that the resulting fibers have high stiffness and resistance to fracture, besides being very strongly anchored to the substrate. Key research issues which need to be addressed for maturing this process have been presented. Extending this technique to a variety of nonconductive and electroactive polymer fibers, many novel applications in novel micro/nanoscale sensors, actuators, fibrillar structures, and optical and electronic devices would become possible.

\section{ACKNOWLEDGMENT}

The authors would like to thank to Prof. T. Kowalewski and Prof. S. Anna for their valuable insights, help, and resources, B. Aksak for AFM measurements and data analysis, J. Garcia and Prof. Fred II Higgs for nano-indentation experiments and data analysis, Prof. N. Urban for providing micro-pipette probes, and their colleagues in the Nanorobotics and Computational Fluid Dynamics and Heat Transfer Laboratories for the valuable comments and suggestions.

\section{REFERENCES}

[1] M. R. Stan, P. D. Franzon, S. C. Goldstein, J. C. Lach, and M. W. Ziegler, "Molecular electronics: from devices and interconnect to circuits and architecture," Proc. IEEE, vol. 91, no. 11, pp. 1940-1957, Nov. 2003.
[2] X. Wang, C. Drew, L. Soo-Hyoung, K. J. Senecal, J. Kumar, and L. A. Samuelson, "Electrospun nanofibrous membranes for highly sensitive optical sensors," Nano Lett., vol. 2, pp. 1273-1275, 2002.

[3] J. Baliga, "Chips go vertical," IEEE Spectrum Mag., vol. 41, no. 3, pp. 43-47, Mar. 2004.

[4] M. C. McAlpine, R. S. Friedman, S. Jin, K. Lin, W. U. Wang, and C. M. Lieber, "High-performance nanowire electronics and photonics on glass and plastic substrates," NanoLett., vol. 3, no. 11, pp. 1531-1535, 2003.

[5] K. Autumn, M. Sitti, Y. A. Liang, A. M. Peattie, W. R. Hansen, S. Sponberg, T. W. Kenny, R. Fearing, J. N. Israelachvili, and R. Full, "Evidence for van der Waals adhesion in gecko setae," in Proc. PNAS, 2002, vol. 99, no. 19 , pp. 12252-12256.

[6] E. Matta and R. P. Tytus, "Liquid stretching using a falling cylinder," J. Non-Newtonian Fluid Mechanics, vol. 35, pp. 215-229, 1990.

[7] S. L. Anna and G. H. McKinley, "Elasto-capillary thinning and breakup of model elastic liquids," J. Rheol., vol. 45, no. 1, pp. 115-138, 2001.

[8] D. H. Reneker and I. Chun, "Nanometre diameter fibres of polymer produced by electrospinning," Nanotechnology, vol. 7, no. 3, pp. 216-223, 1996.

[9] D. H. Reneker, A. L. Yarin, H. Fong, and Koombhongse, "Bending instability of electrically charged liquid jets of polymer solutions in electrospinning," J. Appl. Phys., vol. 87, no. 9, pp. 4531-4547, 2000.

[10] J. M. Deitzel, J. Kleinmeyer, D. Harris, and N. C. Tan, "The effect of processing variables on the morphology of electrospun nanofibers and textiles," Polymer, vol. 42, pp. 261-272, 2001.

[11] M. Bognitzki, W. Czado, T. Frese, A. Schaper, M. Hellwig, M. Steinhart, A. Greiner, and H. Wendorff, "Nanostructured fibers via electrospinning," Adv. Mater., vol. 13, no. 1, pp. 70-72, 2001.

[12] E. Zussman, A. Theron, and A. Yarin, "Formation of nanofiber crossbars in electrospinning," Appl. Phys. Lett., vol. 82, no. 6, pp. 973-975, 2003.

[13] D. Li and Y. Xia, "Electrospinning of nanofibers: reinventing the wheel?," Adv. Mater., vol. 16, no. 14, pp. 1151-1170, 2004.

[14] Z. Huang, Y. Zhang, M. Kotaki, and S. Ramakrishna, "A review on polymer nanofibers by electrospinning and their applications in nanocomposites," Composites Sci. Technol., vol. 63, pp. 2223-2253, 2003.

[15] J. Doshi and D. H. Reneker, "Electrospinning process and application of electrospun fibers," J. Electrostatics, vol. 35, pp. 151-156, 1995.

[16] A. Theron, E. Zussman, and A. Yarin, "Electrostatic field-assisted alignment of electrospun nanofibres," Nanotechnology, vol. 12, pp. 384-390, 2001.

[17] J. Deitzel, J. D. Kleinmeyer, J. K. Hirvonen, and N. C. Tan, "Controlled deposition of electrospun poly(ethylene oxide) fibers," Polymer, vol. 42, no. 19, pp. 8163-8170, 2001.

[18] H. Fong, D. Liu, S. Wang, and R. A. Vaia, "Generation of electrospun fibers of nylon 6 and nylon 6-montmorillonite nanocomposite," Polymer, vol. 43, no. 3, pp. 775-780, 2002.

[19] R. Dersch, T. Liu, A. K. Schaper, A. Greiner, and J. H. Wendorff, "Electrospun nanofibers: internal structure and intrinsic orientation," J. Polymer Sci., vol. 41, no. 4, pt. A, pp. 545-553, 2003.

[20] D. Li, Y. Wang, and Y. Xia, "Electrospinning of polymeric and ceramic nanofibers as uniaxially aligned arrays," Nano Lett., vol. 3, pp. $1167-1171,2003$.

[21] B. Sundary, V. Subramanian, T. S. Natarajan, R. Z. Xiang, C. C. Chang, and W. S. Fann, "Electrospinning of continuous aligned polymer fibers," Appl. Phys. Lett., vol. 84, no. 7, pp. 1222-1224, 2004.

[22] T. Ondarcuhu and C. Joachim, "Drawing a single nanofiber over hundreds of microns," Europhys. Lett., vol. 42, pp. 215-218, 1998.

[23] A. Nain and M. Sitti, "3-D nano-fiber manufacturing by controlled pulling of liquid polymers using nano-probes," in Proc IEEE ConfNanotechnology, 2003, vol. 1, pp. 60-63.

[24] A. Nain, D. H. Goldman, and M. Sitti, "Three-dimensional nanoscale manipulation and manufacturing using proximal probes: controlled pulling of polymer micro/nanofibers," in Proc. IEEE Int. Conf. Robotics and Automation, 2004, vol. 1, pp. 434-439.

[25] A. Nain, C. Amon, and M. Sitti, "Three-dimensional nanoscale manipulation and manufacturing using proximal probes: controlled pulling of polymer micro/nanofibers," in Proc. IEEE Conf. Mechatronics, 2004, pp. 224-230. 
[26] S. A. Harfenist, S. D. Cambron, R. S. Keynton, and R. W. Cohn, "Custom fabrication of freestanding and suspended three-dimensional polymer structures," in Proc. IEEE Conf. Nanotechnology, 2003, vol. 2 , pp. 557-560.

[27] S. A. Harfenist, S. D. Cambron, E. W. Nelso, S. M. Berry, A. W. Isham, M. M. Crain, K. M. Walsh, R. S. Keynton, and R. W. Cohn, "Direct drawing of suspended filamentary micro- and nanostructures from liquid polymers," Nano Lett., vol. 4, no. 10, pp. 1931-1937, 2004.

[28] M. Yao and G. H. McKinley, "Numerical simulation of extensional deformations of viscoelastic liquid bridges in filament stretching devices," J. Non-Newtonian Fluid Mech., vol. 74, pp. 47-88, 1998.

[29] R. Bird, R. C. Armstrong, and O. Hassager, "Dynamics of polymeric liquids," in Fluid Mechanics, 2nd ed. New York: Wiley, 1987.

[30] M. Yao, G. H. McKinley, and B. Debbaut, "Extensional deformation, stress relaxation and necking failure of viscoelastic filaments," J. NonNewtonian Fluid Mech., vol. 79, pp. 469-501, 1998.

[31] A. Tripathi, P. Whittingstall, and G. H. McKinley, "Using filament stretching rheometry to predict strand formation and "processability" in adhesives and other non-Newtonian fluids," Rheol. Acta, vol. 39, pp. 321-337, 2000.

[32] Z. Gou and A. J. McHugh, "A comparison of Newtonian and viscoelastic constitutive models for dry spinning of polymer fibers," $J$. Appl. Polymer Sci., vol. 87, no. 13, pp. 2136-2145, 2003.

[33] A. Pedicini and R. J. Farris, "Mechanical behavior of electrospun polyurethane," Polymer, vol. 44, pp. 6857-6862, 2003.

[34] C. Jiang, M. G. Kuzyk, J.-L. Ding, W. E. Johns, and D. J. Welker, "Fabrication and mechanical behavior of dye-doped polymer optical fiber," J. Appl. Physics, vol. 92, no. 1, pp. 4-12, 2002.

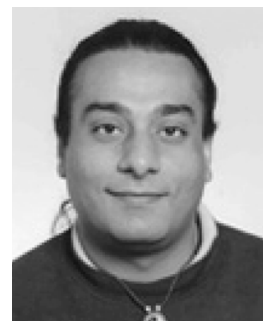

Amrinder Singh Nain received the B.Sc. degree in mechanical engineering from Manipal Institute of Technology, Manipal, India, in 1990). He completed his graduate studies in mechanical engineering at the University of Rhode Island, Kingston, in 1996 and Yale University, New Haven, CT, in 2000. He is currently working toward the Ph.D. degree in mechanical engineering and is involved in fabricating polymer micro/nanofibers and understanding the material behavior at reduced lengthscales.

He has considerable experience working in the industry and has been employed as a Eutectic Engineer by Larsen\&Toubro (L\&T), Mumbai, India from 1990 to 1993, and as Senior Staff Engineer specializing in robotics/precision engineering by Silicon Valley Group Lithography Systems (now part of ASML), Wilton, CT, from 1996 to 2000.

Mr. Nain is recipient of 2002 Dowd-ICES Graduate Student Fellowship and was the finalist for best paper at IEEE International Conference on Robotics and Automation (ICRA) 2004.

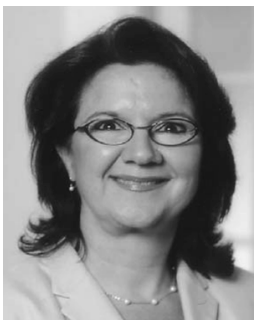

Cristina Amon (SM'95-F'02) received the engineering degree from Universidad Simon Bolivar, Venezuela. She received the M.S. and Ph.D. degrees in mechanical engineering from the Massachusetts Institute of Technology, Cambridge in 1981 and 1988, respectively.

She is the Director of the Institute for Complex Engineered Systems and Raymond J. Lane Distinguished Professor at Carnegie Mellon University, Pittsburgh, PA. Her pioneering CFD research has advanced portable electronics cooling technologies, nanoscale thermal and bioengineering. She has contributed 12 book chapters, one custom textbook, and over 250 refereed articles in the education and research literature.

Dr. Amon is the recipient of numerous awards for excellent in research and education, including best paper awards, ASEE George Westinghouse award (1997), SWE Distinguished Engineering Educator (1999), ASME Gustus Larson Memorial Medal (2000), ASEE Ralph Coats Roe award (2002), Hispanic Engineer Achievement (2003) and ASME EPPD Thermal Management award, for Outstanding Contributions in Thermal Management Applications to the Field of Electronic and Photonic Packaging (2004). She has served as chair of the ASME HTD K-16 Committee on Electronics Cooling (2000-2003) and executive member of the ASME Electronic and Photonic Packaging Division. She was elected General Chair of the 2002 IEEE/ASME ITherm and Program Chair of the 2005 InterPACK/Heat Transfer conference. Her editorship roles include associate editor for the ASME Journal of Heat Transfer, IEEE TRANSACTIONS ON COMPONENTS AND PACKaging TeChnOlOgY, Associate Editor for Electronic Packaging G\&B Book Series, and co-editor of the Journal Heat and Mass Transfer. She is a Fellow of AAAS, ASEE, and ASME and member of the National Academy of Engineering.

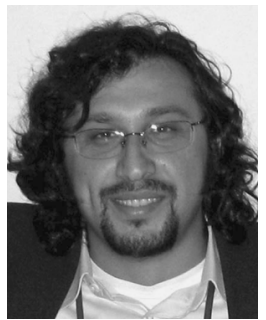

Metin Sitti (S'99-M'99) received the B.Sc. and M.Sc. degrees in electrical and electronics engineering from Bogazici University, Istanbul, Turkey, in 1992 and 1994, respectively, and the Ph.D. degree in electrical engineering from the University of Tokyo, Tokyo, Japan, in 1999.

He was a Research Scientist and Lecturer in the Department of Electrical Engineering and Computer Sciences, University of California at Berkeley during 1999-2002. He is currently an Assistant Professor in Department of Mechanical Engineering with courtesy appointments in Robotics Institute, Electrical and Computer Engineering, and Biomedical Engineering at Carnegie Mellon University, Pittsburgh, PA. He is the Director of the NanoRobotics Laboratory. His research interests include biologically inspired miniature robots and micro/nanoscale manipulation and manufacturing systems.

Dr. Sitti received the National Science Foundation CAREER award and the CMU Struminger award in 2005. He was elected as the Distinguished Lecturer of the IEEE Robotics and Automation Society for 2006-2008. He received the best biomimetics paper award in the IEEE Robotics and Biomimetics Conference (2004), the best paper award in the IEEE/RSJ International Conference on Intelligent Robots and Systems (1998), and the best video award (2002) in the IEEE Robotics and Automation Conference. He is the chair of the IEEE Nanotechnology Council, Nanorobotics and Nanomanufacturing Technical Committee and the co-chair of the IEEE Robotics and Automation Society, Rapid Prototyping in Robotics and Automation Technical Committee. He is a member of the ASME. 